\title{
Simultaneous Hypothesis Testing of Multivariable Nonparametric Spline Regression in the GWR Model
}

\author{
Sifriyani \\ Correspondence: Sifriyani, Departement of Mathematics, Faculty of Mathematics and Natural Sciences, Mulawarman \\ University, Samarinda Indonesia. E-mail: sifriyanistatistika@gmail.com
}

Received: May 19, 2019 Accepted: June 17, 2019 Online Published: June 25, 2019

doi:10.5539/ijsp.v8n4p32 URL: https://doi.org/10.5539/ijsp.v8n4p32

\begin{abstract}
In this research, studied multivariable nonparametric geographically weighted regression use truncated spline approach. The model is an expansion of nonparametric truncated spline regression that takes into account geographical or spatial factors. The purpose of this study was to find statistics test and distribution for the simultaneous hypothesis test. This study obtains the statistic test used the maximum likelihood ratio test (MLRT) method. Results of the research obtained statistics test based on the ratio between the maximum of the likelihood function under the set of $\mathrm{H}_{0}$ and the maximum of the set likelihood function below the population with each have a spatial factor. Distribution of statistical tests has been proven to have a distribution of $\mathrm{F}$. The modeling application used the percentage of the death of Dengue Hemorrhagic Fever (DHF) in 38 districts/cities in East Java Province. The modeling resulted in the determination coefficient of $80.7 \%$ and SSE value that is 0.0043 .
\end{abstract}

Keywords: nonparametric regression, truncated spline, geographically weighted regression, multivariable, simultaneous test

\section{Introduction}

Regression analysis is a method in statistics used to model relationship patterns and mathematical models between response variable $(y)$ with predictor variable $(x)$. The method aims to estimate or predict the value of the response variable if the value of the predictor variable is known (Draper and Smith, 1998). One method used to model spatial data is Geographically Weighted Regression (GWR). GWR model contains predictor variables which each regression coefficient depends on the location where the data is observed. Each parameter value is estimated at each observation location, therefore each point of observation location has different parameter values (Fotheringham, et al. 2002). If each parameter is constant at each geographic location, the GWR model will be the same as the linear regression model. This means that each geographic location has the same model. Research using the GWR theory is conducted by Brunsdon, et al (1995, 1996, 1997, 1999), Fotheringham, et al (1995, 1997), Crespo, et al (2007), Leung, et al (2000b).

The GWR method developed is still in linear form. In several realities faced in both spatial and non-spatial data modeling, the question is whether all the relationships between predictor and response variables form a known regression curve, for example linear pattern. In fact, not all data relations patterns have known regression curves. Considering the rapid development of science and technology and paying attention to natural symptoms leading to unusual patterns, it is very difficult to predict natural behavior. In the past decade, a farmer was able to predict exactly when the dry and rainy season began and ended in various geographical regions very well, therefore farmers were able to prepare themselves when starting rice and when to harvest it, but now it is difficult. The problem of the percentage of poverty, underdevelopment, literacy rates, increasing ignorance, and uneven development in each region along with the causal variables are some examples of events whose pattern is unclear and does not follow a certain pattern hence as to be irregular (Budiantara, 2009).

GWR has been developed to address the problem of spatial heterogeneity. But a good model should be viewed from various aspects and put a modeling problem right on the portion. The differences in environmental and geographical characteristics between observation locations result in observations having different variations, or there are differences in the influence of predictor variables on the response variables for each observation location. How do you solve if the influence of the predictor variable on the response variable does not follow a certain pattern and there are changing patterns in certain sub-sub intervals? In this situation the GWR model has not been able to overcome this problem, therefore it is highly considered that the researchers developed a nonparametric regression in the GWR model (Sifriyani et al, 2018c). 
The purpose of this study was to find a statistical simultaneous test for nonparametric regression parameters in the GWR model using the Maximum Likelihood Ratio Test method. The purpose of the next study, found the distribution of test statistics and decision-making criteria. After obtaining the formulation from the statistical test, it was then applied to Dengue Fever Data in 38 Regencies / Cities in East Java.

\section{Multivariable Nonparametric Spline Regression in the GWR Model}

Multivariable Nonparametric Spline Regression in the GWR Model is the development of nonparametric regression for spatial data with parameter estimators of a local nature for each observation location (Sifriyani et all, 2018c). In the regression model the assumption used is an error with a normal distribution with zero mean and variance $\sigma^{2}\left(u_{i}, v_{i}\right)$ at each $\left(u_{i}, v_{i}\right)$ location. Location coordinates $\left(u_{i}, v_{i}\right)$ are one of the important factors in determining the weight used to estimate the parameters of the model. Data is given $\left(x_{1 i}, x_{2 i}, \ldots, x_{l i}, y_{i}\right)$ and the relationship between $\left(x_{1 i}, x_{2 i}, \ldots, x_{l i}\right)$ and $y_{i}$ is assumed to follow the multivariable nonparametric regression model as follows:

$$
y_{i}=f\left(x_{1 i}, x_{2 i}, \ldots, x_{l i}\right)+\varepsilon_{i}, \quad i=1,2, \ldots, n,
$$

where $y_{i}$ as the response variable and $f\left(x_{1 i}, x_{2 i}, \ldots, x_{l i}\right)$ is a regression curve that is unknown and assumed to be additive.

Mathematically the form of the relationship between the response variable $y_{i}$ and the predictor variable $\left(x_{1 i}, x_{2 i}, \ldots, x_{l i}\right)$ at the $i$ location for the Multivariable Nonparametric Spline Regression in the GWR Model, can be stated as follows (Sifriyani et all, 2018a):

$$
y_{i}=\beta_{0}\left(u_{i}, v_{i}\right)+\sum_{p=1}^{l} \sum_{k=1}^{m} \beta_{p k}\left(u_{i}, v_{i}\right) x_{p i}^{k}+\sum_{p=1}^{l} \sum_{h=1}^{r} \delta_{p, m+h}\left(u_{i}, v_{i}\right)\left(x_{p i}-K_{p h}\right)_{+}^{m}
$$

Equation (2) is a Multivariable Nonparametric Spline Regression model in the GWR Model m degree with $\mathrm{n}$ area. Based on equation (2), simultaneous hypothesis testing will be carried out using the maximum likelihood ratio test.

\section{Methods}

The steps to determine the hypothesis for simultaneous test of Multivariable Nonparametric Geographically Weighted Regression Use Truncated Spline Approach are as follows:

Step 1. Applying hypothesis model

$$
\begin{gathered}
H_{0}: \beta_{11}\left(u_{i}, v_{i}\right)=\beta_{12}\left(u_{i}, v_{i}\right)=\cdots=\beta_{l m}\left(u_{i}, v_{i}\right)=\delta_{1, m+1}\left(u_{i}, v_{i}\right)= \\
\delta_{1, m+2}\left(u_{i}, v_{i}\right)=\cdots=\delta_{l, m+r}\left(u_{i}, v_{i}\right)=0, i=1,2, \ldots, n \\
H_{1}: \text { at least there is one of } \beta_{p k}\left(u_{i}, v_{i}\right) \neq 0 \text { or } \delta_{p, m+h}\left(u_{i}, v_{i}\right) \neq 0, \\
\quad p=1,2, \ldots, l ; k=1,2, \ldots, m ; h=1,2, \ldots, r ; i=1,2, \ldots, n
\end{gathered}
$$

Step 2. Defining the parameters set under the population $\boldsymbol{\Omega}$.

Step 3. Obtaining the MLE estimator for the parameters under $\boldsymbol{\Omega}$.

Step 4. Maximizing the likelihood function under $\boldsymbol{\Omega}$ namely $L(\hat{\Omega})$.

Step 5. Defining the parameter space under $\mathrm{H}_{0}$ namely $\omega$.

Step 6. Determining estimator $\hat{\beta}_{0}\left(u_{i}, v_{i}\right)$ and $\hat{\sigma}_{\omega}^{2}\left(u_{i}, v_{i}\right)$ which are parameters under $\mathrm{H}_{0}$.

Step 7. Obtaining maximum likelihood function under $\mathrm{H}_{0}$ namely $L(\hat{\omega})$.

Step 8. Obtaining the statistic test

Step 9. Determining the distribution of $\frac{\tilde{\mathrm{Y}}^{\mathrm{T}} M\left(u_{i}, v_{i}\right) \tilde{\mathrm{Y}}}{\sigma^{2}} \sim \frac{\chi_{(\operatorname{tr}((\mathrm{I}-\xi)(\mathrm{I}-\xi)))^{2}}^{2}}{\sum_{i=1}^{n} \gamma_{i}^{2}}$

Step 10. Determining the distribution of $\frac{\tilde{\mathrm{Y}}^{\mathrm{T}} D\left(u_{i}, v_{i}\right) \tilde{\mathrm{Y}}}{\sigma^{2}\left(\frac{\sum_{i=1}^{n} \lambda_{i}^{2}}{\operatorname{tr}\left((\mathrm{I}-\xi)^{\mathrm{T}}(\mathrm{I}-\xi)\right)}\right)} \sim \chi_{\left(\frac{\left(\operatorname{tr}\left((\mathrm{I}-\xi)^{\mathrm{T}}(\mathrm{I}-\xi)\right)\right)^{2}}{\sum_{i=1}^{n} \lambda_{i}^{2}}\right)}^{2}$

Step 10. Determining the distribution of statistic test $V^{*}$.

Step 11. Determining the rejection area of $H_{0}$ 


\section{Step 12. Empirical study}

\section{Result and Discussion}

4.1 Parameter Estimation Under Hypothesis $H_{0}$ and $H_{1}$ For Multivariable Nonparametric Geographically Weighted Regression Use Truncated Spline Approach

After obtaining the result of hypothesis testing which stated that the Multivariable Nonparametric Geographically Weighted Regression Use Truncated Spline Approach is not the same as the nonparametric truncated spline regression model (Sifriyani, 2018b). Further research is to perform simultaneous test of parameters for Multivariable Nonparametric Geographically Weighted Regression Use Truncated Spline Approach with hypothetical form (2). Futhermore, given parameter space under $\mathrm{H}_{0}$ which is symbolized by $L(\varpi)$ and parameter space under population which is symbolized by $L(\Omega)$. The set of parameters under $\mathrm{H}_{0}$ ie:

$$
\begin{array}{r}
\varpi=\left\{\beta_{0}\left(u_{i}, v_{i}\right), \beta_{11}\left(u_{i}, v_{i}\right), \ldots, \beta_{l m}\left(u_{i}, v_{i}\right), \delta_{1 m+1}\left(u_{i}, v_{i}\right), \ldots, \delta_{l m+r}\left(u_{i}, v_{i}\right) \mid \beta_{11}\left(u_{i}, v_{i}\right)=\right. \\
\left.\beta_{21}\left(u_{i}, v_{i}\right)=\cdots=\beta_{l m}\left(u_{i}, v_{i}\right)=\delta_{1 m+1}\left(u_{i}, v_{i}\right)=\cdots=\delta_{l m+r}\left(u_{i}, v_{i}\right)=0, \sigma_{\omega}^{2}\left(u_{i}, v_{i}\right)\right\}
\end{array}
$$

Lemma 1 and Lemma 2 are given to explain the parameter estimator of model.

\section{Lemma 1}

If $\beta_{0}\left(u_{i}, v_{i}\right)$ is parameter under $\mathrm{H}_{0}$ from equation (2), then the estimator $\hat{\beta}_{0}\left(u_{i}, v_{i}\right)$ is given by:

$$
\hat{\beta}_{0}\left(u_{i}, v_{i}\right)=\frac{\sum_{j=1}^{n} w_{j(i)} y_{j}}{\sum_{j=1}^{n} w_{j(i)}}
$$

\section{Proof}

To obtain the estimator $\hat{\beta}_{0}\left(u_{i}, v_{i}\right)$, we form a likelihood function under parameter space of population $L(\varpi)$. Since $y_{i}$ is normal distributed with mean $\beta_{0}\left(u_{i}, v_{i}\right)$ and variance $\sigma_{\varpi}^{2}\left(u_{i}, v_{i}\right)$, then the probability functions of $y_{1}, y_{2}, \ldots, y_{n}$ are given by:

$$
f\left(y_{1}, y_{2}, \ldots, y_{n}\right)=\prod_{j=1}^{n}\left\{\frac{1}{\sqrt{2 \pi \sigma_{\varpi}^{2}\left(u_{i}, v_{i}\right)}} \exp \left(-\frac{1}{2 \sigma_{\varpi}^{2}\left(u_{i}, v_{i}\right)}\left[y_{j}-\beta_{0}\left(u_{i}, v_{i}\right)\right]^{2}\right)\right\}
$$
Furthermore, the joint probability function above is assigned a geographical weighting $w_{j(i)}$ to obtain a likelihood
function as follows:

$$
L(\varpi)=(2 \pi)^{-\frac{n}{2}}\left(\sigma_{\varpi}^{2}\left(u_{i}, v_{i}\right)\right)^{-\frac{n}{2}} \exp \left(-\frac{1}{2 \sigma_{\varpi}^{2}\left(u_{i}, v_{i}\right)} \sum_{j=1}^{n} w_{j(i)}\left[y_{j}-\beta_{0}\left(u_{i}, v_{i}\right)\right]^{2}\right)
$$

Ln likelihood function is given by:

$$
\begin{aligned}
& \ln L(\varpi)=-\frac{n}{2} \ln (2 \pi)-\frac{n}{2} \ln \left(\sigma_{\varpi}^{2}\left(u_{i}, v_{i}\right)\right)-\frac{1}{2 \sigma_{\varpi}^{2}\left(u_{i}, v_{i}\right)} \\
& \sum_{j=1}^{n}\left(w_{j(i)} y_{j}^{2}-2 w_{j(i)} y_{j} \beta_{0}\left(u_{i}, v_{i}\right)+w_{j(i)}\left(\beta_{0}\left(u_{i}, v_{i}\right)\right)^{2}\right)
\end{aligned}
$$

Estimator $\hat{\beta}_{0}\left(u_{i}, v_{i}\right)$ will be obtained based on derivative result $\frac{\partial \ln L(\varpi)}{\partial \beta_{0}\left(u_{i}, v_{i}\right)}=0$ as follows:

$$
\frac{\partial \ln L(\varpi)}{\partial \beta_{0}\left(u_{i}, v_{i}\right)}=\frac{1}{\sigma_{\varpi}^{2}\left(u_{i}, v_{i}\right)} \sum_{j=1}^{n} w_{j(i)} y_{j}-\frac{1}{\sigma_{\varpi}^{2}\left(u_{i}, v_{i}\right)} \sum_{j=1}^{n} w_{j(i)} \hat{\beta}_{0}\left(u_{i}, v_{i}\right)
$$

and the estimator $\hat{\beta}_{0}\left(u_{i}, v_{i}\right)$ 


$$
\hat{\beta}_{0}\left(u_{i}, v_{i}\right)=\frac{\sum_{j=1}^{n} w_{j(i)} y_{j}}{\sum_{j=1}^{n} w_{j(i)}}, \quad i=1,2, \ldots, n
$$

Equation (9) is equivalent to equation as follows:

$$
\hat{\tilde{\beta}}_{0}\left(u_{i}, v_{i}\right)=B_{\omega} \tilde{Y}
$$

where

$$
B_{\omega}=\left[\begin{array}{cccc}
\frac{w_{1(1)}}{\sum_{j=1}^{n} w_{j(1)}} & \frac{w_{2(1)}}{\sum_{j=1}^{n} w_{j(1)}} & \cdots & \frac{w_{n(1)}}{\sum_{j=1}^{n} w_{j(1)}} \\
\frac{w_{1(2)}}{\sum_{j=1}^{n} w_{j(2)}} & \frac{w_{2(2)}}{\sum_{j=1}^{n} w_{j(2)}} & \cdots & \frac{w_{n(2)}}{\sum_{j=1}^{n} w_{j(2)}} \\
\vdots & \vdots & \ddots & \vdots \\
\frac{w_{1(n)}}{\sum_{j=1}^{n} w_{j(n)}} & \frac{w_{2(n)}}{\sum_{j=1}^{n} w_{j(n)}} & \cdots & \frac{w_{n(n)}}{\sum_{j=1}^{n} w_{j(n)}}
\end{array}\right], \quad j=1,2, \ldots, n \text { and } \tilde{\mathrm{Y}}=\left[\begin{array}{c}
y_{1} \\
y_{2} \\
\vdots \\
y_{n}
\end{array}\right]
$$

Furthermore, the estimator $\hat{\sigma}_{\sigma}^{2}\left(u_{i}, v_{i}\right)$ is presented in Lemma 2.

\section{Lemma 2}

If $\sigma_{\omega}^{2}\left(u_{i}, v_{i}\right)$ is a parameter under $\mathrm{H} 0$ from equation (2), then the estimator $\hat{\sigma}_{\omega}^{2}\left(u_{i}, v_{i}\right)$ obtained from likelihood function:

$$
L(\varpi)=(2 \pi)^{-\frac{n}{2}}\left(\sigma_{\varpi}^{2}\left(u_{i}, v_{i}\right)\right)^{-\frac{n}{2}} \exp \left(-\frac{1}{2 \sigma_{\varpi}^{2}\left(u_{i}, v_{i}\right)} \sum_{j=1}^{n}\left[y_{j}-\beta_{0}\left(u_{i}, v_{i}\right)\right]^{2}\right)
$$

is given by:

$$
\hat{\sigma}_{\sigma}^{2}\left(u_{i}, v_{i}\right)=\frac{\left(\tilde{\mathrm{Y}}-\hat{\beta}_{0}\left(u_{i}, v_{i}\right)\right)^{\mathrm{T}}\left(\tilde{\mathrm{Y}}-\hat{\beta}_{0}\left(u_{i}, v_{i}\right)\right)}{n}
$$

\section{Proof}

Estimator $\hat{\sigma}_{\sigma}^{2}\left(u_{i}, v_{i}\right)$ is obtsained using likelihood function:

$$
L(\varpi)=(2 \pi)^{-\frac{n}{2}}\left(\sigma_{\varpi}^{2}\left(u_{i}, v_{i}\right)\right)^{-\frac{n}{2}} \exp \left(-\frac{1}{2 \sigma_{\varpi}^{2}\left(u_{i}, v_{i}\right)} \sum_{j=1}^{n}\left[y_{j}-\beta_{0}\left(u_{i}, v_{i}\right)\right]^{2}\right)
$$

Ln likelihood function is given by:

$$
\ln L(\varpi)=-\frac{n}{2} \ln (2 \pi)-\frac{n}{2} \ln \left(\sigma_{\varpi}^{2}\left(u_{i}, v_{i}\right)\right)-\frac{1}{2 \sigma_{\varpi}^{2}\left(u_{i}, v_{i}\right)} \sum_{j=1}^{n}\left[y_{j}-\beta_{0}\left(u_{i}, v_{i}\right)\right]^{2}
$$

maximized against $\hat{\sigma}_{\sigma}^{2}\left(u_{i}, v_{i}\right)$ then obtained:

$$
\frac{\partial \ln L(\varpi)}{\partial \sigma_{\varpi}^{2}\left(u_{i}, v_{i}\right)}=-\frac{n}{2 \hat{\sigma}_{\varpi}^{2}\left(u_{i}, v_{i}\right)}+\frac{1}{2\left(\hat{\sigma}_{\varpi}^{2}\left(u_{i}, v_{i}\right)\right)^{2}} \sum_{j=1}^{n}\left(y_{j}-\beta_{0}\left(u_{i}, v_{i}\right)\right)^{2}
$$


Therefore obtained estimator $\hat{\sigma}_{\sigma}^{2}\left(u_{i}, v_{i}\right)$ ie:

$$
\hat{\sigma}_{\sigma}^{2}\left(u_{i}, v_{i}\right)=\frac{\tilde{\mathrm{Y}}^{\mathrm{T}}\left(\mathrm{I}-B_{\omega}\right)^{\mathrm{T}}\left(\mathrm{I}-B_{\omega}\right) \tilde{\mathrm{Y}}}{n}
$$

Based on Lemma 1 and Lemma 2 obtained the maximum likelihood function as follows:

$$
\begin{aligned}
L(\hat{\omega})= & L\left(\hat{\beta}_{0}\left(u_{i}, v_{i}\right), \hat{\sigma}_{\varpi}^{2}\left(u_{i}, v_{i}\right)\right) \\
= & (2 \pi)^{-\frac{n}{2}}\left(\hat{\sigma}_{\sigma}^{2}\left(u_{i}, v_{i}\right)\right)^{-\frac{n}{2}} \\
& \exp \left(-\frac{1}{2 \hat{\sigma}^{2}\left(u_{i}, v_{i}\right)}\left(\tilde{\mathrm{Y}}-\hat{\beta}_{0}\left(u_{i}, v_{i}\right)\right)^{\mathrm{T}}\left(\tilde{\mathrm{Y}}-\hat{\beta}_{0}\left(u_{i}, v_{i}\right)\right)\right) \\
& =(2 \pi)^{-\frac{n}{2}}\left(\hat{\sigma}_{\sigma}^{2}\left(u_{i}, v_{i}\right)\right)^{-\frac{n}{2}} \exp \left(-\frac{n}{2}\right)
\end{aligned}
$$

Furthermore, given the parameter space under population $(\Omega)$ as follows:

$$
\Omega=\left\{\beta_{11}\left(u_{i}, v_{i}\right), \beta_{21}\left(u_{i}, v_{i}\right), \cdots, \beta_{l m}\left(u_{i}, v_{i}\right), \delta_{1, m+1}\left(u_{i}, v_{i}\right), \cdots, \delta_{l, m+r}\left(u_{i}, v_{i}\right), \sigma_{\Omega}^{2}\left(u_{i}, v_{i}\right)\right\}
$$

and likelihood function on parameter space under hypothesis $\mathrm{H} 1(\Omega)$ ie

$$
\begin{gathered}
L(\Omega)=(2 \pi)^{-\frac{n}{2}}\left(\sigma_{\Omega}^{2}\left(u_{i}, v_{i}\right)\right)^{-\frac{n}{2}} \exp \left(-\frac{1}{2 \sigma_{\Omega}^{2}\left(u_{i}, v_{i}\right)}\right. \\
\left.\sum_{j=1}^{n}\left[y_{j}-\left(\beta_{0}\left(u_{i}, v_{i}\right)+\sum_{p=1}^{l} \sum_{k=1}^{m} \beta_{p k}\left(u_{i}, v_{i}\right) x_{p i}^{k}+\sum_{p=1}^{l} \sum_{h=1}^{r} \delta_{p, m+h}\left(u_{i}, v_{i}\right)\left(x_{p i}-K_{p h}\right)_{+}^{m}\right)\right]^{2}\right)
\end{gathered}
$$

Therefore, the result of $L(\hat{\Omega})$ :

$$
L(\hat{\Omega})=(2 \pi)^{-\frac{n}{2}}\left(\hat{\sigma}_{\Omega}^{2}\left(u_{j}, v_{j}\right)\right)^{-\frac{n}{2}} \exp \left(-\frac{n}{2}\right)
$$

where

$$
\hat{\sigma}_{\Omega}^{2}\left(u_{i}, v_{i}\right)=\frac{\left(\tilde{\mathrm{Y}}-Q \hat{\tilde{\eta}}_{\Omega}\left(u_{i}, v_{i}\right)\right)^{\mathrm{T}}\left(\tilde{\mathrm{Y}}-Q \hat{\tilde{\eta}}_{\Omega}\left(u_{i}, v_{i}\right)\right)}{n}
$$

Futhermore, given Lemma 3 to explain likelihood ratio between $L(\hat{\varpi})$ and $L(\hat{\Omega})$.

\section{Lemma 3}

If $L(\hat{\omega})$ and $L(\hat{\Omega})$ are resspectively given by (18) and (20) then the likelihood ratio $\Lambda$ is given by:

$$
\Lambda^{*}=\frac{\tilde{\mathrm{Y}}^{\mathrm{T}} M\left(u_{i}, v_{i}\right) \tilde{\mathrm{Y}}}{\tilde{\mathrm{Y}}^{\mathrm{T}} D\left(u_{i}, v_{i}\right) \tilde{\mathrm{Y}}}
$$

where

$$
M\left(u_{i}, v_{i}\right)=\left(\mathrm{I}-B_{\omega}\right)^{\mathrm{T}}\left(\mathrm{I}-B_{\omega}\right)
$$




$$
D\left(u_{i}, v_{i}\right)=\left(I-\mathrm{W}\left(u_{i}, v_{i}\right) Q\left(Q^{T} \mathrm{~W}\left(u_{i}, v_{i}\right) Q\right)^{-1} Q^{T}\right)\left(I-Q\left(Q^{T} \mathrm{~W}\left(u_{i}, v_{i}\right) Q\right)^{-1} Q^{T} \mathrm{~W}\right)
$$

\section{Proof}

Based on Lemma 2, the likelihood ratio from equation (17) and (22) sie:

$$
\begin{gathered}
\Lambda^{*}=\frac{L(\hat{\omega})}{L(\hat{\Omega})} \\
=\frac{(2 \pi)^{-\frac{n}{2}}\left(\hat{\sigma}_{\sigma}^{2}\left(u_{i}, v_{i}\right)\right)^{-\frac{n}{2}} \exp \left(-\frac{n}{2}\right)}{(2 \pi)^{-\frac{n}{2}}\left(\hat{\sigma}_{\Omega}^{2}\left(u_{i}, v_{i}\right)\right)^{-\frac{n}{2}} \exp \left(-\frac{n}{2}\right)} \\
=\left(\frac{\hat{\sigma}_{\omega}^{2}\left(u_{i}, v_{i}\right)}{\left.\hat{\sigma}_{\Omega}^{2}\left(u_{i}, v_{i}\right)\right)^{-\frac{n}{2}}}\right. \\
=\left(\begin{array}{c}
\tilde{\mathrm{Y}}^{\mathrm{T}}\left(\mathrm{I}-B_{\omega}\right)^{\mathrm{T}}\left(\mathrm{I}-B_{\omega}\right) \tilde{\mathrm{Y}} \\
\tilde{\mathrm{Y}}^{\mathrm{T}}\left(\mathrm{I}-\mathrm{W}\left(u_{i}, v_{i}\right) Q\left(Q^{\mathrm{T}} \mathrm{W}\left(u_{i}, v_{i}\right) Q\right)^{-1} Q^{\mathrm{T}}\right)\left(\mathrm{I}-Q\left(Q^{\mathrm{T}} \mathrm{W}\left(u_{i}, v_{i}\right) Q\right)^{-1} Q^{\mathrm{T}} \mathrm{W}\left(u_{i}, v_{i}\right)\right) \tilde{\mathrm{Y}}
\end{array}\right)^{-\frac{n}{2}} \\
=\left(\frac{\tilde{\mathrm{Y}}^{\mathrm{T}} M\left(u_{i}, v_{i}\right) \tilde{\mathrm{Y}}}{\tilde{\mathrm{Y}}^{\mathrm{T}} D\left(u_{i}, v_{i}\right) \tilde{\mathrm{Y}}}\right)^{-\frac{n}{2}} \mathbf{\square}
\end{gathered}
$$

4.2 Statistic Test for Multivariable Nonparametric Geographically Weighted Regression Use Truncated Spline Approach

Given the following simultaneous test of parameters for multivariable nonparametric truncated spline regression in the GWR model, which is complete given by Theorem 4.

\section{Theorem 4}

If likelihood ratio $\Lambda$ is given by Lemma 3 then the simultaneous test of parameters for multivariable nonparametric truncated spline regression in the GWR model on equation (1) is given by:

$$
V^{*}=\frac{\frac{\left(\tilde{\mathrm{Y}}^{\mathrm{T}} M\left(u_{i}, v_{i}\right) \tilde{\mathrm{Y}}\right.}{\left(\frac{\left.\left.\operatorname{tr}\left(\mathrm{I}-B_{\omega}\right)^{\mathrm{T}}\left(\mathrm{I}-B_{\omega}\right)\right)\right)^{2}}{\sum_{i=1}^{n} \gamma_{i}^{2}}\right)}}{\frac{\tilde{\mathrm{Y}}^{\mathrm{T}} D\left(u_{i}, v_{i}\right) \tilde{\mathrm{Y}}}{\left(\operatorname{tr}\left((\mathrm{I}-\xi)^{\mathrm{T}}(\mathrm{I}-\xi)\right)\right)^{2}}}
$$

\section{Proof}

Based on Lemma 3 obtained likelihood ratio as follows:

$$
\Lambda=\left(\frac{\tilde{\mathrm{Y}}^{\mathrm{T}} M\left(u_{i}, v_{i}\right) \tilde{\mathrm{Y}}}{\tilde{\mathrm{Y}}^{\mathrm{T}} D\left(u_{i}, v_{i}\right) \tilde{\mathrm{Y}}}\right)^{-\frac{n}{2}}
$$

Based in MLRT method, hypothesis $\mathrm{ssH}_{0}$ is rejected if:

$$
\Lambda=\left(\frac{\tilde{\mathrm{Y}}^{\mathrm{T}} M\left(u_{i}, v_{i}\right) \tilde{\mathrm{Y}}}{\tilde{\mathrm{Y}}^{\mathrm{T}} D\left(u_{i}, v_{i}\right) \tilde{\mathrm{Y}}}\right)^{-\frac{n}{2}}<c
$$

For a constant $c$. Equation (27) is equivalent to: 


$$
\begin{aligned}
& \tilde{\mathrm{Y}}^{\mathrm{T}} D\left(u_{i}, v_{i}\right) \tilde{\mathrm{Y}}<c^{\frac{2}{n}} \tilde{\mathrm{Y}}^{\mathrm{T}} M\left(u_{i}, v_{i}\right) \tilde{\mathrm{Y}} \\
& \frac{1}{c^{\frac{2}{n}}}<\frac{\tilde{\mathrm{Y}}^{\mathrm{T}} M\left(u_{i}, v_{i}\right) \tilde{\mathrm{Y}}}{\tilde{\mathrm{Y}}^{\mathrm{T}} D\left(u_{i}, v_{i}\right) \tilde{\mathrm{Y}}} \\
& \left(\frac{\tilde{\mathrm{Y}}^{\mathrm{T}} M\left(u_{i}, v_{i}\right) \tilde{\mathrm{Y}}}{\tilde{\mathrm{Y}}^{\mathrm{T}} D\left(u_{i}, v_{i}\right) \tilde{\mathrm{Y}}}\right)>c^{-\frac{2}{n}}
\end{aligned}
$$

The two sections of the inequality above, the numerator are each divided by:

$$
\frac{\left(\operatorname{tr}\left(\left(\mathrm{I}-B_{\omega}\right)^{\mathrm{T}}\left(\mathrm{I}-B_{\omega}\right)\right)\right)^{2}}{\sum_{i=1}^{n} \gamma_{i}^{2}}
$$

And each denominator is divided by:

$$
\frac{\left(\operatorname{tr}\left((\mathrm{I}-\xi)^{\mathrm{T}}(\mathrm{I}-\xi)\right)\right)^{2}}{\sum_{i=1}^{n} \lambda_{i}^{2}}
$$

Then the inequality is obtained:

$$
\left.\frac{\frac{\tilde{\mathrm{Y}}^{\mathrm{T}} M\left(u_{i}, v_{i}\right) \tilde{\mathrm{Y}}}{\left.\frac{\left(\operatorname{tr}\left(\left(\mathrm{I}-B_{\omega}\right)^{\mathrm{T}}\left(\mathrm{I}-B_{\omega}\right)\right)\right)^{2}}{\sum_{i=1}^{n} \gamma_{i}^{2}}\right)}}{\frac{\tilde{\mathrm{Y}}^{\mathrm{T}} D\left(u_{i}, v_{i}\right) \tilde{\mathrm{Y}}}{\left(\frac{\left(\operatorname{tr}\left((\mathrm{I}-\xi)^{\mathrm{T}}(\mathrm{I}-\xi)\right)\right)^{2}}{\sum_{i=1}^{n} \lambda_{i}^{2}}\right)}}>c^{-\frac{2}{n}}\right) \frac{\left(\frac{\left(\operatorname{tr}\left((\mathrm{I}-\xi)^{\mathrm{T}}(\mathrm{I}-\xi)\right)\right)^{2}}{\sum_{i=1}^{n} \lambda_{i}^{2}}\right)}{\left(\frac{\left(\operatorname{tr}\left(\left(\mathrm{I}-B_{\omega}\right)^{\mathrm{T}}\left(\mathrm{I}-B_{\omega}\right)\right)\right)^{2}}{\sum_{i=1}^{n} \gamma_{i}^{2}}\right)}=c^{*}
$$

Consequently the statistic test of hypothesis $\mathrm{H}_{0}$ and $\mathrm{H}_{1}$ is given by:

$$
V^{*}=\frac{\left.\frac{\tilde{\mathrm{Y}}^{\mathrm{T}} M\left(u_{i}, v_{i}\right) \tilde{\mathrm{Y}}}{\sum_{i=1}^{n} \gamma_{i}^{2}}\right)}{\frac{\left(\operatorname{tr}\left((\mathrm{I}-\xi)^{\mathrm{T}}(\mathrm{I}-\xi)\right)\right)^{2}}{D\left(u_{i}, v_{i}\right) \tilde{\mathrm{Y}}}}
$$

The resulting statistical test is different from the results of Leung et all, 2000, Fotheringham, et al 1995 and Fotheringham, et al 1997. Equation (23) contains the knot point and the nature of the idempotent matrix, so that for the completion of the distribution of statistics test cannot use several theories which said that if $\boldsymbol{A}$ is a symmetric matrix sized $n \times n$ and random vector $\boldsymbol{y}$ has $N(\mathbf{0}, \boldsymbol{I})$ distribution hence $\boldsymbol{y}^{T} \boldsymbol{A} \boldsymbol{y}$ follows a $\chi_{r}^{2}$ distribution if and only if $\boldsymbol{A}$ is an idempotent matrix and has rank $r$ (Rencher, 2000). 


\subsection{Distribution of Statistic Test and Critical Area of Parameter Hypothesis for Model}

Next we find the distribution of the statisticaltest $V^{*}$. To obtain the distribution of this statistic test, Theorem 5 is required.

\section{Theorem5}

If $M\left(u_{i}, v_{i}\right)$ is matrix given by Lemma 2 then the statistics:

$$
\frac{\tilde{\mathrm{Y}}^{\mathrm{T}} M\left(u_{i}, v_{i}\right) \tilde{\mathrm{Y}}}{\sigma^{2}} \sim \frac{\chi_{\left(\operatorname{tr}\left((\mathrm{I}-\xi)^{\mathrm{T}}(\mathrm{I}-\xi)\right)\right)^{2}}^{2}}{\sum_{i=1}^{n} \gamma_{i}^{2}}
$$

\section{Proof}

Based on Lemma 1, obtained

$$
\hat{\tilde{Y}}=B_{\omega} \tilde{Y}
$$

Based on the equation above, obtained vector of error as follows:

$$
\hat{\tilde{\varepsilon}}=\tilde{\mathrm{Y}}-\hat{\tilde{\mathrm{Y}}}=\left(\mathrm{I}-B_{\omega}\right) \tilde{\mathrm{Y}}
$$

Furthermore the sum squareof error $(\mathrm{JKE})$ under $\mathrm{H}_{0}$ is obtained by squaring the following vector of error:

$$
\mathrm{JKE}=\hat{\widetilde{\varepsilon}}^{\mathrm{T}} \hat{\tilde{\varepsilon}}=\tilde{\mathrm{Y}}^{\mathrm{T}}\left(\mathrm{I}-B_{\omega}\right)^{\mathrm{T}}\left(\mathrm{I}-B_{\omega}\right) \tilde{\mathrm{Y}}
$$

where $E(\hat{\tilde{\varepsilon}})=\beta_{0}\left(u_{i}, v_{i}\right)-E\left(\hat{\beta}_{0}\left(u_{i}, v_{i}\right)\right)=\tilde{0}$ dan $E\left(\tilde{\varepsilon} \tilde{\varepsilon}^{\mathrm{T}}\right)=\sigma^{2} \mathrm{I}$

Obtained sum square of error under $\mathrm{H}_{0}$ :

$$
\begin{gathered}
\mathrm{JKE}=(\tilde{\mathrm{Y}}-\hat{\tilde{\mathrm{Y}}})^{\mathrm{T}}(\tilde{\mathrm{Y}}-\hat{\tilde{\mathrm{Y}}}) \\
=\tilde{\varepsilon}^{\mathrm{T}}\left(\mathrm{I}-B_{\omega}\right)^{\mathrm{T}}\left(\mathrm{I}-B_{\omega}\right) \tilde{\varepsilon}
\end{gathered}
$$

$\left(\mathrm{I}-B_{\omega}\right)^{\mathrm{T}}\left(\mathrm{I}-B_{\omega}\right)$ is a symmetric matrix and $\tilde{\varepsilon} \sim N\left(\tilde{0}, \sigma^{2} \mathrm{I}\right)$, then the expected value of equation (11) is:

$$
\begin{gathered}
E(\mathrm{JKE})=E\left(\operatorname{tr}\left(\tilde{\varepsilon}^{\mathrm{T}}\left(\mathrm{I}-B_{\omega}\right)^{\mathrm{T}}\left(\mathrm{I}-B_{\omega}\right) \tilde{\varepsilon}\right)\right) \\
=\operatorname{tr}\left(\left(\mathrm{I}-B_{\omega}\right)^{\mathrm{T}}\left(\mathrm{I}-B_{\omega}\right)\right) \sigma^{2}
\end{gathered}
$$

Since $\tilde{\varepsilon}^{\mathrm{T}}\left(\mathrm{I}-B_{\omega}\right)^{\mathrm{T}}\left(\mathrm{I}-B_{\omega}\right) \tilde{\varepsilon}$ is a quadratic form of random variables:

$$
\tilde{\mathrm{Y}}^{\mathrm{T}}\left(\mathrm{I}-B_{\omega}\right)^{\mathrm{T}}\left(\mathrm{I}-B_{\omega}\right) \tilde{\mathrm{Y}}>0
$$

where $\left(\mathrm{I}-B_{\omega}\right)^{\mathrm{T}}\left(\mathrm{I}-B_{\omega}\right)$ is a symmetric matrix sized $n \times n$. Consequently the matrix $\left(\mathrm{I}-B_{\omega}\right)^{\mathrm{T}}\left(\mathrm{I}-B_{\omega}\right)$ is positive semi definite, but matrix $\left(\mathrm{I}-B_{\omega}\right)^{\mathrm{T}}\left(\mathrm{I}-B_{\omega}\right)$ is not idempotent. Futhermore, obtained:

$$
\frac{\mathrm{JKE}}{\sigma^{2}}=\frac{\tilde{\varepsilon}^{\mathrm{T}}}{\sigma}\left(\mathrm{I}-B_{\omega}\right)^{\mathrm{T}}\left(\mathrm{I}-B_{\omega}\right) \frac{\tilde{\varepsilon}}{\sigma}
$$

Since $\tilde{\varepsilon} \sim N\left(\tilde{0}, \sigma^{2} \mathrm{I}\right)$ therefore $\frac{\tilde{\varepsilon}}{\sigma} \sim N(\tilde{0}, \mathrm{I})$. Then, since matrix $\left(\mathrm{I}-B_{\omega}\right)^{\mathrm{T}}\left(\mathrm{I}-B_{\omega}\right)$ is not idempotent, the distribution of statistics is as follows: 


$$
\frac{\mathrm{JKE}}{\sigma^{2}} \sim c \chi_{p}^{2}
$$

For constants $c$ and $p$. Based on equation (12), obtained:

$$
E\left(\frac{\mathrm{JKE}}{\sigma^{2}}\right)=\operatorname{tr}\left(\left(\mathrm{I}-B_{\omega}\right)^{\mathrm{T}}\left(\mathrm{I}-B_{\omega}\right)\right)
$$

Since $\left(\mathrm{I}-B_{\omega}\right)^{\mathrm{T}}\left(\mathrm{I}-B_{\omega}\right)$ is a symmetric and positive semi definite matrix then based on Theorem 2 on Chapter III, there was an orthogonal matrix $H$, hence:

$$
H\left(\mathrm{I}-B_{\omega}\right)^{\mathrm{T}}\left(\mathrm{I}-B_{\omega}\right) H^{\mathrm{T}}=\Lambda=\operatorname{diag}\left(\gamma_{1}, \gamma_{2}, \ldots, \gamma_{n}\right)
$$

With $\Lambda$ is a diagonal matrix which $\gamma_{1}, \gamma_{2}, \ldots, \gamma_{n}$ are Eigen values from matrix $\left(\mathrm{I}-B_{\omega}\right)^{\mathrm{T}}\left(\mathrm{I}-B_{\omega}\right)$. The result obtained:

$$
\begin{aligned}
\frac{\mathrm{JKE}}{\sigma^{2}} & =\frac{\tilde{\varepsilon}^{\mathrm{T}}}{\sigma}\left(\mathrm{I}-B_{\omega}\right)^{\mathrm{T}}\left(\mathrm{I}-B_{\omega}\right) \frac{\tilde{\varepsilon}}{\sigma} \\
& =\frac{\tilde{\varepsilon}^{\mathrm{T}}}{\sigma} \Lambda \frac{\tilde{\varepsilon}}{\sigma} \\
& =\sum_{i=1}^{n} \gamma_{i}\left(\frac{\tilde{\varepsilon}_{i}}{\sigma}\right)^{2}
\end{aligned}
$$

where $\Lambda=H\left(\mathrm{I}-B_{\omega}\right)^{\mathrm{T}}\left(\mathrm{I}-B_{\omega}\right) H^{\mathrm{T}}, \quad \tilde{\varepsilon}=\left[\begin{array}{llll}\varepsilon_{1} & \varepsilon_{2} & \cdots & \varepsilon_{n}\end{array}\right]^{\mathrm{T}}$ and random variables $\frac{\tilde{\varepsilon}_{1}}{\sigma}, \frac{\tilde{\varepsilon}_{2}}{\sigma}, \ldots, \frac{\tilde{\varepsilon}_{n}}{\sigma}$ are normal distributed which is independent and identical. Hence:

$$
\left(\frac{\tilde{\varepsilon}_{i}}{\sigma}\right)^{2} \sim \chi_{1}^{2}
$$

With mean 1 and variance 2. Hence:

$$
\begin{aligned}
\operatorname{var}\left(\frac{\mathrm{JKE}}{\sigma^{2}}\right) & =\operatorname{var}\left(\sum_{i=1}^{n} \gamma_{i}\left(\frac{\tilde{\varepsilon}_{i}}{\sigma}\right)^{2}\right) \\
& =2 \sum_{i=1}^{n} \gamma_{i}^{2}
\end{aligned}
$$

Since $\frac{\tilde{\varepsilon}^{\mathrm{T}}\left(\mathrm{I}-B_{\omega}\right)^{\mathrm{T}}\left(\mathrm{I}-B_{\omega}\right) \tilde{\varepsilon}}{\sigma^{2}} \sim c \chi_{p}^{2}$ therefore:

$$
\begin{aligned}
E\left(\frac{\mathrm{JKE}}{\sigma^{2}}\right) & =\operatorname{tr}\left(\left(\mathrm{I}-B_{\omega}\right)^{\mathrm{T}}\left(\mathrm{I}-B_{\omega}\right)\right)=c p \\
\operatorname{var}\left(\frac{\mathrm{JKE}}{\sigma^{2}}\right) & =2 \sum_{i=1}^{n} \gamma_{i}^{2} \\
& =2 c^{2} p
\end{aligned}
$$

The values of $\mathrm{c}$ and $\mathrm{p}$ are substituted in the following equation:

$$
\begin{aligned}
2 c^{2} p & =2 \sum_{i=1}^{n} \gamma_{i}^{2} \\
p & =\frac{\left(\operatorname{tr}\left(\left(\mathrm{I}-B_{\omega}\right)^{\mathrm{T}}\left(\mathrm{I}-B_{\omega}\right)\right)\right)^{2}}{\sum_{i=1}^{n} \gamma_{i}^{2}} \quad \text { and } \quad c=\frac{\sum_{i=1}^{n} \gamma_{i}^{2}}{\operatorname{tr}\left(\left(\mathrm{I}-B_{\omega}\right)^{\mathrm{T}}\left(\mathrm{I}-B_{\omega}\right)\right)}
\end{aligned}
$$


Thus the distribution of

$$
\frac{\mathrm{JKE}}{c \sigma^{2}}=\frac{\left(\operatorname{tr}\left(\left(\mathrm{I}-B_{\omega}\right)^{\mathrm{T}}\left(\mathrm{I}-B_{\omega}\right)\right)\right)^{2}}{\sum_{i=1}^{n} \gamma_{i}^{2}} \frac{\hat{\sigma}^{2}}{\sigma^{2}} \sim \chi_{p}^{2}
$$

This shows that $\frac{\mathrm{JKE}}{\sigma^{2}}$ follows the distribution of $c \chi_{p}^{2}$ with mean $c p$ variance $2 c^{2} p$ and the degree of freedom $\frac{\left(\operatorname{tr}\left(\left(\mathrm{I}-B_{\omega}\right)^{\mathrm{T}}\left(\mathrm{I}-B_{\omega}\right)\right)\right)^{2}}{\sum_{i=1}^{n} \gamma_{i}^{2}}$.

Given the JKE of equation (10) and equation (15), obtained

$$
\frac{\tilde{\mathrm{Y}}^{\mathrm{T}} M\left(u_{i}, v_{i}\right) \tilde{\mathrm{Y}}}{\sigma^{2}} \sim \frac{\chi^{2}\left(\operatorname{tr}\left(\left(\mathrm{I}-B_{\omega}\right)^{\mathrm{T}}\left(\mathrm{I}-B_{\omega}\right)\right)\right)^{2}}{\sum_{i=1}^{n} \gamma_{i}^{2}} \mathbf{\square}
$$

\section{Corollary 6}

If statistics $V^{*}$ is given by Theosrem 4 , therefore

$$
\left.V^{*}=\frac{\frac{\left(\frac{\left(\operatorname{tr}\left(\left(\mathrm{I}-B_{\omega}\right)^{\mathrm{T}}\left(\mathrm{I}-B_{\omega}\right)\right)\right)^{2}}{\sum_{i=1}^{n} \gamma_{i}^{2}}\right)}{\left(\frac{\left(\operatorname{tr}\left((\mathrm{I}-\xi)^{\mathrm{T}}(\mathrm{I}-\xi)\right)\right)^{2}}{\sum_{i=1}^{n} \lambda_{i}^{2}}\right)} \sim\left(\frac{\tilde{\mathrm{Y}}^{\mathrm{T}} D\left(u_{i}, v_{i}\right) \tilde{\mathrm{Y}}}{\left.\left.\left(\operatorname{tr}-B_{\omega}\right)^{\mathrm{T}}\left(\mathrm{I}-B_{\omega}\right)\right)\right)^{2}}\right.}{\sum_{i=1}^{n} \gamma_{i}^{2}}, \frac{\left(\operatorname{tr}\left((\mathrm{I}-\xi)^{\mathrm{T}}(\mathrm{I}-\xi)\right)\right)^{2}}{\sum_{i=1}^{n} \lambda_{i}^{2}}\right)
$$

\section{Proof}

Based on Theorem 5, obtained statistics:

$$
\frac{\tilde{\mathrm{Y}}^{\mathrm{T}} M\left(u_{i}, v_{i}\right) \tilde{\mathrm{Y}}}{\sigma^{2}} \sim \frac{\chi_{\left(\operatorname{tr}\left(\left(\mathrm{I}-B_{\omega}\right)^{\mathrm{T}}\left(\mathrm{I}-B_{\omega}\right)\right)\right)^{2}}^{2}}{\sum_{i=1}^{n} \gamma_{i}^{2}}
$$

Furthermore, obtained statistics:

$$
\tau^{*}=\frac{\tilde{\mathrm{Y}}^{\mathrm{T}} D\left(u_{i}, v_{i}\right) \tilde{\mathrm{Y}}}{\sigma^{2}} \sim \frac{\chi_{\left.\left(\mathrm{tr}(\mathrm{I}-\xi)^{\mathrm{T}}(\mathrm{I}-\xi)\right)\right)^{2}}^{2}}{\sum_{i=1}^{n} \lambda_{i}^{2}}
$$

As a result, obtained statistics: 


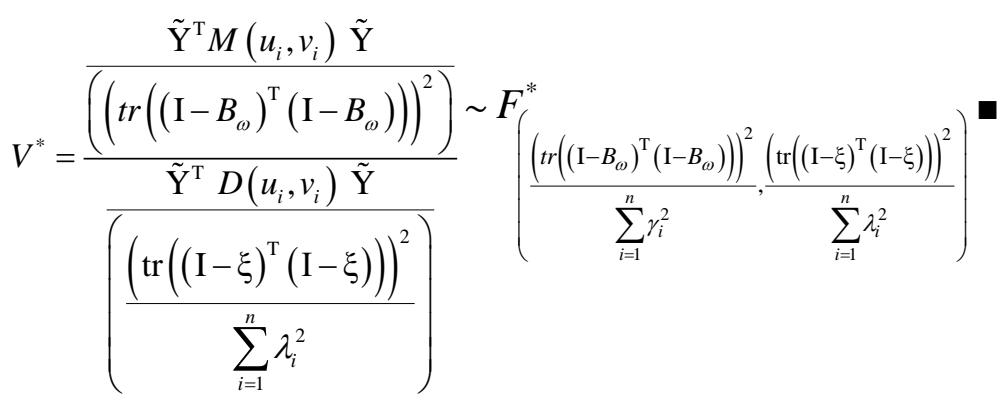

Here is derived the rejection area for simultaneous hypothesis of parameters in multivariable nonparametric truncated spline regression in the GWR model, the critical area for this test is given by Lemma 7.

\section{Lemma 7}

If given a test statistic $V^{*}$ as in Theorem 4 then the rejection area for hypothesis $\mathrm{H}_{0}$ in Equation (2) is given by:

$$
C\left(y, x_{1}, x_{2}, \ldots, x_{l}\right)=\left\{\left(y, x_{1}, x_{2}, \ldots, x_{l}\right) ; V^{*}>c\right\}
$$

For a constant $\mathrm{c}$ obtained by the equation:

$$
P\left(V^{*}>c\right)=\alpha, \quad 0<\alpha<1
$$

where $\alpha$ is a level of significance which is determined and

$$
V^{*} \sim F^{*}\left(\frac{\left(\operatorname{tr}\left(\left(\mathrm{I}-B_{\omega}\right)^{\mathrm{T}}\left(\mathrm{I}-B_{\omega}\right)\right)\right)^{2}}{\sum_{i=1}^{n} \gamma_{i}^{2}}, \frac{\left(\mathrm{tr}\left((\mathrm{I}-\xi)^{\mathrm{T}}(\mathrm{I}-\xi)\right)\right)^{2}}{\sum_{i=1}^{n} \lambda_{i}^{2}}\right)
$$

\section{Proof}

Based on Theorem 4, obtained:

$$
\left.\frac{\frac{\tilde{\mathrm{Y}}^{\mathrm{T}} M\left(u_{i}, v_{i}\right) \tilde{\mathrm{Y}}}{\left.\frac{\left(\operatorname{tr}\left(\left(\mathrm{I}-B_{\omega}\right)^{\mathrm{T}}\left(\mathrm{I}-B_{\omega}\right)\right)\right)^{2}}{\sum_{i=1}^{n} \gamma_{i}^{2}}\right)}}{\frac{\tilde{\mathrm{Y}}^{\mathrm{T}} D\left(u_{i}, v_{i}\right) \tilde{\mathrm{Y}}}{\left(\frac{\left(\operatorname{tr}\left((\mathrm{I}-\xi)^{\mathrm{T}}(\mathrm{I}-\xi)\right)\right)^{2}}{\sum_{i=1}^{n} \lambda_{i}^{2}}\right)}} c^{-\frac{2}{n}}\right)=c^{*}
$$

For a constant $c^{*}$ and based on Corollary 6 and equation 16, then the level of significance $\alpha$ given by hypothesis $\mathrm{H}_{0}$ is rejected if

$$
V^{*}>F^{*}\left(\frac{\left(\operatorname{tr}\left(\left(\mathrm{I}-B_{\omega}\right)\left(\mathrm{I}-B_{\omega}\right)\right)\right)^{2}}{\sum_{i=1}^{n} \gamma_{i}^{2}}, \frac{\left(\mathrm{tr}\left((\mathrm{I}-\xi)^{\mathrm{T}}(\mathrm{I}-\xi)\right)\right)^{2}}{\sum_{i=1}^{n} \lambda_{i}^{2}}\right) \text { - }
$$

\subsection{Empirical Study on Percentage of Death of Dengue Hemorrhagic Fever (DHF)}

\section{Response Variables}

Response variable used in this research is percentage of death of Dengue Hemorrhagic Fever (DHF) in 38 districts/cities in East Java Province.

\section{Predictor Variables}

Predictor variables in this study are the variables that are suspected to affect the number of DHF sufferers in 38 
districts/cities in the East Java Province. The predictor variables used are the percentage of health facilities $\left(x_{1}\right)$, percentage of health personnel $\left(x_{2}\right)$, percentage of poor people $\left(x_{3}\right)$ and average rainfall $\left(x_{4}\right)$.

Simultaneous Hypothesis Test

Hypothesis

The hypotheses for simultaneous significance testing are as follows:

$H_{0}: \beta_{11}\left(u_{i}, v_{i}\right)=\beta_{12}\left(u_{i}, v_{i}\right)=\cdots=\beta_{42}\left(u_{i}, v_{i}\right)=0$

and $\delta_{13}\left(u_{i}, v_{i}\right)=\delta_{14}\left(u_{i}, v_{i}\right)=\cdots=\delta_{44}\left(u_{i}, v_{i}\right)=0$

$H_{1}$ : At least there is one of $\beta_{p k}\left(u_{i}, v_{i}\right) \neq 0$ or $\delta_{p, m+h}\left(u_{i}, v_{i}\right) \neq 0$,

$p=1,2,3,4 ; k=1,2 ; h=1,2 ; i=1,2, \ldots, 38$

Test Statistic

To see the four predictor variables that simultaneously affect the percentage of deaths of DHF in 38 districts/cities in East Java Province, we use the test statistic in Theorem 4 as follows:

$$
V^{*}=\frac{\left(\frac{\tilde{\mathrm{Y}}^{\mathrm{T}} M\left(u_{i}, v_{i}\right) \tilde{\mathrm{Y}}}{\operatorname{tr}\left(\left(\mathrm{I}-B_{\omega}\right)^{\mathrm{T}}\left(\mathrm{I}-B_{\omega}\right)\right)}\right)}{\left(\frac{\tilde{\mathrm{Y}}^{\mathrm{T}} D\left(u_{i}, v_{i}\right) \tilde{\mathrm{Y}}}{\operatorname{tr}\left((\mathrm{I}-\xi)^{\mathrm{T}}(\mathrm{I}-\xi)\right)}\right)}
$$

The calculation result for the numerator of Equation (17) is as follows:

$$
\left(\frac{\tilde{\mathrm{Y}}^{\mathrm{T}} M\left(u_{i}, v_{i}\right) \tilde{\mathrm{Y}}}{\operatorname{tr}\left(\left(\mathrm{I}-B_{\omega}\right)^{\mathrm{T}}\left(\mathrm{I}-B_{\omega}\right)\right)}\right)=3,2429
$$

The denominator of Equation (7) is as follows:

$$
\left(\frac{\tilde{\mathrm{Y}}^{\mathrm{T}} D\left(u_{i}, v_{i}\right) \tilde{\mathrm{Y}}}{\operatorname{tr}\left((\mathrm{I}-\xi)^{\mathrm{T}}(\mathrm{I}-\xi)\right)}\right)=1,8356
$$

Hence, the test statistic $V^{*}=1,7667$.

The rejection area for the simultaneous test of multivariable nonparametric truncated spline regression in the GWR model is given by Lemma 7. By using the level of significance $\alpha=0,05$, concluded that $\mathrm{H} 0$ is rejected since $V^{*}=1,7667>\mathrm{F}(0,05 ; 36,27)=1,60$. It is concluded that there is at least one parameter in the multivariable nonparametric truncated spline regression in the GWR model that is significant to the response variable.

Table 1 shows the comparison of modeling using OLS, GWR, Nonparametric Spline (TSR) and Nonparametric Truncated Spline Regression in the GWR Models (GWR-TSR).

Table 1. Comparison of OLS, TSR, GWR and GWR-TSR Models

\begin{tabular}{c|r|r|r|r}
\hline Criteria & \multicolumn{1}{|c|}{ OLS } & \multicolumn{1}{c|}{ GWR } & \multicolumn{1}{c}{ TSR } & \multicolumn{1}{c}{ GWR-TSR } \\
\hline$R^{2}$ & $54,1 \%$ & $72,3 \%$ & $65,5 \%$ & $80,7 \%$ \\
\hline SSE & 0,0286 & 0,0067 & 0,0089 & 0,0043 \\
\hline
\end{tabular}

Based on Table 1, the modeling of the percentage of deaths of DHF sufferers in 38 districts/cities in East Java Province using (GWR-TSR) model is better than OLS, GWR and TSR models. This can be seen from the smallest SSE value that is 0.0043 and $\mathrm{R}^{2}=80.7 \%$ which means that the model is able to explain the data of $80.7 \%$.

\section{Conclusion}

Hypothesis test for parameters of multivariable nonparametric truncated spline regression in the GWR models uses the hypothesis formula as follows:

$$
\begin{aligned}
H_{0}: \beta_{11}\left(u_{i}, v_{i}\right) & =\beta_{12}\left(u_{i}, v_{i}\right)=\cdots=\beta_{l m}\left(u_{i}, v_{i}\right)=\delta_{1, m+1}\left(u_{i}, v_{i}\right)=\delta_{1, m+2}\left(u_{i}, v_{i}\right)=\cdots=\quad \delta_{l, m+r}\left(u_{i}, v_{i}\right)=0, i \\
& =1,2, \ldots, n
\end{aligned}
$$

$H_{1}$ : At least there is one of $\beta_{p k}\left(u_{i}, v_{i}\right) \neq 0$ atau $\delta_{p, m+h}\left(u_{i}, v_{i}\right) \neq 0$, 
$p=1,2, \ldots, l ; k=1,2, \ldots, m ; h=1,2, \ldots, r ; i=1,2, \ldots, n$

In deriving the test statistics and the distribution using the Maximum Likelihood Ratio Test (MLRT) method, it was obtained:

1. Based on estimator $\hat{\tilde{\eta}}_{\Omega}\left(u_{i}, v_{i}\right)$ and $\hat{\sigma}_{\Omega}^{2}\left(u_{i}, v_{i}\right)$, obtained maximum likelihood function under population $(\Omega)$ :

$$
\begin{gathered}
L(\hat{\Omega})=(2 \pi)^{-\frac{n}{2}}\left(\hat{\sigma}_{\Omega}^{2}\left(u_{j}, v_{j}\right)\right)^{-\frac{n}{2}} \exp \left(-\frac{n}{2}\right) \\
\text { where } \hat{\sigma}_{\Omega}^{2}\left(u_{i}, v_{i}\right)=\frac{\left(\tilde{\mathrm{Y}}-Q \hat{\tilde{\eta}}_{\Omega}\left(u_{i}, v_{i}\right)\right)^{\mathrm{T}}\left(\tilde{\mathrm{Y}}-Q \hat{\tilde{\eta}}_{\Omega}\left(u_{i}, v_{i}\right)\right)}{n}
\end{gathered}
$$

and maximum likelihood function under hypothesis $\mathrm{H}_{0}(\varpi)$ :

$$
L(\hat{\varpi})=(2 \pi)^{-\frac{n}{2}}\left(\left(\tilde{\mathrm{Y}}-B_{\omega} \tilde{\mathrm{Y}}\right)^{\mathrm{T}}\left(\tilde{\mathrm{Y}}-B_{\omega} \tilde{\mathrm{Y}}\right)\right)^{-\frac{n}{2}} \exp \left(-\frac{n}{2}\right)
$$

2. Test statistic for simultaneous test of parameters of multivariable nonparametric truncated spline regression in the GWR models:

$$
V^{*}=\frac{\left.\frac{\tilde{\mathrm{Y}}^{\mathrm{T}} M\left(u_{i}, v_{i}\right) \tilde{\mathrm{Y}}}{\sum_{i=1}^{n} \gamma_{i}^{2}}\right)}{\frac{\left(\operatorname{tr}\left(\left(\mathrm{I}-B_{\omega}\right)^{\mathrm{T}}\left(\mathrm{I}-B_{\omega}\right)\right)\right)^{2}}{D\left(u_{i}, v_{i}\right) \tilde{\mathrm{Y}}}}
$$

3. The distribution of test statistic for simultaneous test of parameters of multivariable nonparametric truncated spline regression in the GWR models:

$$
V^{*} \sim F_{\left(\frac{\left.\operatorname{tr}\left(\left(\mathrm{I}-B_{\omega}\right)^{\mathrm{T}}\left(\mathrm{I}-B_{\omega}\right)\right)\right)^{2}}{\sum_{i=1}^{n} \gamma_{i}^{2}}, \frac{\left(\operatorname{tr}\left((\mathrm{I}-\xi)^{\mathrm{T}}(\mathrm{I}-\xi)\right)\right)^{2}}{\sum_{i=1}^{n} \lambda_{i}^{2}}\right)}
$$

4. The rejection area for hypothesis $\mathrm{H}_{0}$ :

$$
C\left(y, x_{1}, x_{2}, \ldots, x_{l}\right)=\left\{\left(y, x_{1}, x_{2}, \ldots, x_{l}\right) ; V^{*}>c\right\}
$$

For a constant c obtained by equation:

$$
P\left(V^{*}>c\right)=\alpha, \quad 0<\alpha<1
$$

where $\alpha$ is a level of significance which is determined and

$$
V^{*} \sim F^{*}\left(\frac{\left(\operatorname{tr}\left(\left(\mathrm{I}-B_{\omega}\right)^{\mathrm{T}}\left(\mathrm{I}-B_{\omega}\right)\right)\right)^{2}}{\sum_{i=1}^{n} \gamma_{i}^{2}}, \frac{\left(\operatorname{tr}\left((\mathrm{I}-\xi)^{\mathrm{T}}(\mathrm{I}-\xi)\right)\right)^{2}}{\sum_{i=1}^{n} \lambda_{i}^{2}}\right)
$$




\section{Acknowledgements}

The author acknowledge The IsDB Research Grant and Project Implementation Unit for generously supporting this project.

\section{References}

Antoniadis, A., Bigot, J., \& Spatinas, T. (2001). Wavelet Estimators in Nonparametric Regression: A Comparative Simulation Study. Journal of Statistical Software, 6, 1-83. https://doi.org/10.18637/jss.v006.i06

Antoniadis, A., Gregorire, G., \& Mackeagu, W. (1994). Wavelet Methods for Curve Estimation. Journal of the American Statistical Association, 89, 1340-1353. https://doi.org/10.1080/01621459.1994.10476873

Baladandayuthapani, M., \& Carroll. (2005). Spatially Adaptive Bayesian Regression Splines (P-Spline). Journal of Computational and Graphical Statistics, 14, 378-394. https://doi.org/10.1198/106186005X47345

Biau, \& Cadre. (2004). Nonparametric spatial prediction. Statistics Inference Stochastic Process, 7, $327-349$. https://doi.org/10.1023/B:SISP.0000049116.23705.88

Biau, G. (2003). Spatial kernel density estimation. Mathematics Methods Statistics, 12, 371-390.

Brunsdon, \& Fotheringham. (1999). Some notes on parametric significance test for Geographically Weighted Regression. Journal of Regional Science, 39(3), 497-524. https://doi.org/10.1111/0022-4146.00146

Brunsdon, C. F. (1995). Estimating probability surfaces for geographical point data: An adaptive kernel algorithm. Computers and Geosciences, 21, 877-894. https://doi.org/10.1016/0098-3004(95)00020-9

Brunsdon, C., Fotheringham, A. S., \& Charlton, M. (1997). Geographical Instability in Linear Regression Modelling - A Preliminary Investigation. in IOS Press New Techniques and Technologies for Statistics II, Amsterdam. Oxford and Washington, 149-158.

Brunsdon, F., \& Charlton. (1996). Geograhically weighted regression: a method for exploring spatial nonstationarity. Geographical Analysis, 28, 281-298. https://doi.org/10.1111/j.1538-4632.1996.tb00936.x

Budiantara, I. N. (2001). Aplikasi Spline Estimator Terbobot. Jurnal Teknik Industri, 3(2), 57-62.

Budiantara, I. N. (2005). Model Keluarga Spline Polinomial Truncated Dalam Regresi Semiparametrik. Jurnal berkala ilmiah MIPA. 15.

Budiantara, I. N. (2009). Spline dalam Regresi Nonparametrik dan Semiparametrik, Sebuah Pemodelan Statistika Masa Kini dan Masa Mendatang. Speech of Inauguration of Professor. Institut Tekhnologi Sepuluh Nopember. Surabaya.

Budiantara, I. N., Subanar, \& Soejoeti, Z. (1997). Weighted Spline Estimator. Bulletin of the International Statistical Institute, 51, 333-334.

Crespo, R., Fotheringham, A., \& Charlton, M. (2007). Application of geograhically weighted regression to a 19-year set of house price data in London to calibrate local hedonic price models. Proceeding of the 9th International Conference on Geocomputation.

Draper, \& Smith. (1998). Applied Regression Analysis. John Wiley. New York. https://doi.org/10.1002/9781118625590

Fedele, G., Massimo, V., \& Elisa, C. (2018). P-spline smoothing for spatial data collected worldwide. Spatial Statistics, In press, accepted manuscript. Available online 5 September 2018.

Fotheringham, A. S., \& Pitts, T. C. (1995). Directional Variation in Distance Decay. Environment and Planning A, 27. 715-729. https://doi.org/10.1068/a270715

Fotheringham, A. S., Brundson, C., \& Charlton, M. (2002). Geographically Weighted Regression: The Analysis of Spatially Varying Relationships, John Wiley \& Sons Ltd, England.

Fotheringham, A. S., Charlton, M., \& Brunsdon, C. (1997). Two techniques for exploring non-stationarity in geographical data. Geographical Systems, 4, 59-82.

Fotheringham, A. S., \& CharltonM, B. C. (1997a). 'Measuring Spatial Variations in Relationships with GeographicallyWeighted Regression; in M.M. Fischer and A. Getis eds', Recent Developments in Spatial Analysis, Springer, Berlin/New York, 60-82. https://doi.org/10.1007/978-3-662-03499-6_4

Giannelli, J., \& Speleers. (2012). THB-Spline:The Truncated basis for hierarchical Spline. Computer Aided Geometric Design, 29, 485-498. https://doi.org/10.1016/j.cagd.2012.03.025

Green, P. J., \& Silverman, B. W. (1994). Nonparametric Regression and Generalized Linear Model. Chapman \& Hall: London. https://doi.org/10.1007/978-1-4899-4473-3 
Guillaume, P., Yves, F., Francois, G., Claudine, B., Michel, G., \& Olivier, G. (2011). Nonparametric Spatial Regression of survival probability: Visualization Of Population Sinks In Eurasian Woodcock. Ecological Society of America. 92(8), 1672-1679. https://doi.org/10.1890/10-2224.1

Hardle, G. (1990). Applied Nonparametric Regression. Cambridge University Press: New York. https://doi.org/10.1017/CCOL0521382483

Hien, D. N., Geoffrey, J. M., \& Ian, A. W. (2016). Mixtures of spatial spline regressions for clustering and classification. Computational Statistics and Data Analysis, 93, 79-85. https://doi.org/10.1016/j.csda.2014.01.011

Jiawei, Z., \& Xuefeng. (2006). The Construction of Wavelet-Based Truncated Conical Shell element Using B-Spline Wavelet on The Interval. Acta Mechanica Solida Sinica, 19(4), 316-326. https://doi.org/10.1007/s10338-006-0638-0

Lei-Lei, L., Shao-He, Z., \& Yung-Ming, C. (2018). Advanced reliability analysis of slopes in spatially variable soils using ultivariate adaptive regression spline. Geoscience Frotiers, In press, corrected proof. Available online 9 May 2018.

Leung, Y., Mei, C. L., \& Zhang, W. X. (2000a). Statistic Test for Spatial Non stationarity Based on the Geographically Weighted Regression Model. Journal Environment and Planing A, 32, 9-32. https://doi.org/10.1068/a3162

Leung, Y., Mei, C. L., \& Zhang, W. X. (2000b). Testing for spatial autocorrelation among the residuals of the geograhically weighted regression. Environment and Planing A, 32, 871-890. https://doi.org/10.1068/a32117

Maria, X., Martin, P., Fred, A., \& Paul, H. C. (2018). Correcting for spatial heterogeneity in plant breeding experiments with P-splines, Spatial Statistics, 23, 52-71. https://doi.org/10.1016/j.spasta.2017.10.003

Pintore, S., \& Holmes. (2006). Spatially Adaptive Smoothing Splines. Biometrika, 93, 113-125. https://doi.org/10.1093/biomet/93.1.113

Rencher, A. C. (2000). Linear Model in Statistics. John Wiley \& Sons Inc, Singapore.

Ruppert, D., \& Carroll, R. J. (2000). Spatially-adaptive penalties for spline fitting. Aust. New Zeal. Journal Statistics. 42, 205-23. https://doi.org/10.1111/1467-842X.00119

Sangalli, L. M., Ramsay, J. O., \& Ramsay, T. O. (2013). Spatial spline regression models. Journal Royal Statistics Soc. Ser. B., 75, 681-703. https://doi.org/10.1111/rssb.12009

Sifriyani, B. I. N., Kartiko, S. H., \& Gunardi. (2019). Evaluation of Factors Affecting Increased Unemployment in East Java Using NGWR-TS Method. International Journal of Sciences: Basic and Applied Research, 49(1), $123-142$.

Sifriyani, H., Budiantara, I. N., \& Gunardi. (2017). Geographically Weighted Regression with Spline Approach. Far East Journal of Mathematical Sciences, 101(6), 1183-1196. https://doi.org/10.17654/MS101061183

Sifriyani, I. N., Budiantara, S. H., Kartiko, \& Gunardi. (2018b). A New Method of Hypothesis Test for Truncated Spline Nonparametric Regression Influenced by Spatial Heterogeneity and Application. Abstract and Applied Analysis. https://doi.org/10.1155/2018/9769150

Sifriyani, S. H., Kartiko, I. N., Budiantara, \& Gunardi. (2018a). Development Of Nonparametric Geographically Weighted Regression Using Truncated Spline Approach. Songklanakarin Journal of Science And Technology, 40(4), 909-920.

Sifriyani. (2018c). Multivariable Nonparametric Regression Truncated Spline in The Geographically Weighted Regression Models, Unpublished Ph.D, Dissertation, Universitas Gadjah Mada, Dept of Mathematics.

Wahba, G. (1990). Spline Models For Observasion Data. SIAM. Pensylvania. https://doi.org/10.1137/1.9781611970128

Zhou, S., \& Shen. (2000). Spatially adaptive regression spline and accurate knot selection schemes. Journal of the American Association, 96, 247-259. https://doi.org/10.1198/016214501750332820

\section{Copyrights}

Copyright for this article is retained by the author(s), with first publication rights granted to the journal.

This is an open-access article distributed under the terms and conditions of the Creative Commons Attribution license (http://creativecommons.org/licenses/by/4.0/). 